\title{
Hybrid total arch repair without deep hypothermic circulatory arrest for acute type A aortic dissection (R1)
}

\author{
Qian Chang, MD, Chuan Tian, MD, Yizhen Wei, MD, Xiangyang Qian, MD, Xiaogang Sun, MD, and \\ Cuntao Yu, MD
}

Objective: To investigate the surgical outcomes of hybrid total arch repair without deep hypothermic circulatory arrest for patients with acute Stanford type A aortic dissection.

\begin{abstract}
Methods: Retrospective review of clinical data of patients with acute Stanford type A aortic dissection who underwent surgical repair at our institution between November 2009 and December 2011 identified 21 patients who underwent hybrid total arch repair without deep hypothermic circulatory arrest. The in-hospital and follow-up data were investigated. Postoperative serial computed tomography angiography was used to evaluate the fate of true and false lumen in arch and descending aorta.
\end{abstract}

Results: Mean follow-up was 13.8 months (range, 3 to 21 months). The 1- and 12-month survival rates (by Kaplan-Meier analysis) were $95.2 \%$ (95\% confidence interval, $86.2 \%-100 \%)$ and $90.5 \%$ (95\% confidence interval, $78.0 \%-100 \%$ ), respectively. No endograft caudal migration occurred. One patient with type I endoleak was successfully resolved during operation. There was no late rupture or paraplegia.

Conclusions: Hybrid total arch repair without deep hypothermic circulatory arrest offers a promising alternative to risk reduction of complications during the postoperative period and late adverse events resulting from false lumen enlargement in the arch and descending aorta. (J Thorac Cardiovasc Surg 2013;146:1393-8)

Conventional surgical procedure (ascending aortic replacement) designed to cure the ascending aortic pathology in acute Stanford type A aortic dissection left patients with untreated residual dissection in aortic arch and distal aorta. ${ }^{1}$ After ascending aortic surgery for Stanford type A aortic dissection, gradual expansion of a false lumen and aneurysmal formation in the aortic arch and the thoracoabdominal aorta remains a clinical challenge. ${ }^{2,3}$ Previous reports ${ }^{4-8}$ describe a surgical procedure of total aortic arch under deep hypothermic circulatory arrest in acute type A aortic dissection and insertion of stent graft into the descending aorta to diminish a false lumen of the aortic arch and descending aorta. Deep hypothermic circulatory arrest could elongate cardiopulmonary bypass duration and lead to a more severe inflammatory response. To avoid deep hypothermic circulatory arrest could possibly provide theoretical organ protection for patients with acute type A aortic dissection.

We explored the clinical use of a single-stage hybrid procedure that combines ascending aortic replacement and endovascular total arch repair in acute Stanford type A aortic

\footnotetext{
From the Department of Cardiovascular Surgery, FuWai Hospital and National Center for Cardiovascular Diseases, Beijing, China.

Disclosures: Authors have nothing to disclose with regard to commercial support. Received for publication March 31, 2012; revisions received July 2, 2012; accepted for publication Sept 13, 2012; available ahead of print Nov 12, 2012.

Address for reprints: Qian Chang, MD, Department of Cardiovascular Surgery, Fu

Wai Hospital and National Center for Cardiovascular Diseases, 167 Bei Li Shi

Rd, West District, Beijing, China 100037 (E-mail: chtfw@hotmail.com). $0022-5223 / \$ 36.00$

Copyright (c) 2013 by The American Association for Thoracic Surgery http://dx.doi.org/10.1016/j.jtcvs.2012.09.041
}

dissection that required no hypothermic circulatory arrest. We investigated the feasibility of such hybrid strategy for acute type A aortic dissection.

\section{METHODS \\ Patient Selection}

We reviewed the clinical data of patients who underwent surgical repair for acute Stanford type A aortic dissection between 2009 and 2011 at Fuwai Hospital and National Center for Cardiovascular Diseases and identified 21patients who underwent a single-stage hybrid total arch repair procedure without deep hypothermic circulatory arrest. Inclusion criteria were Stanford type A aortic dissection with onset within 2 weeks, involvement of the aortic arch and descending aorta, and no previous open cardiac or aortic procedure. Clinical, echocardiographic, serial computed tomographic scanning, operative, and follow-up data were collected for each patient. The study protocol was performed in accordance with the institutional review board, and all patients gave written informed consent for the procedure.

\section{Open Repair Portion (Proximal Ascending Aorta)}

A left radial and femoral arterial line and transesophageal echocardiographic monitoring probe were inserted before general anesthesia was administered. Systemic heparinization $(400 \mathrm{U} / \mathrm{kg})$ was initiated after visualization of innominate, left common carotid, and left subclavian artery through sternotomy. Artery cannulation was placed into the right axillary artery and the right femoral artery. Venous cannulation was inserted through the right atrium. Cardiopulmonary bypass was initiated and rectal temperature was maintained $28^{\circ} \mathrm{C}$.

After crossclamping at the ascending aorta, a longitude incision in the ascending aorta was made and myocardial protection was conducted by antegrade perfusion of cold-blood cardioplegia. All intima dissected in the aortic root and the ascending aorta were resected, and aortic valve commissures were reattached with 4-0 Prolene suture if affected by dissection. After inspection of the morphologic appearance of the cusps and root geometry, and the involvement of the aortic root by the dissection, the 


\section{Abbreviation and Acronym \\ $\mathrm{CTA}=$ computed tomography angiography}

decision for composite aortic root replacement or supracommissural ascending aortic replacement was made. If necessary, the affected sinus of Valsalva was reconstructed with a Dacron graft patch (partial David procedure).

A four-branch Dacron graft (Meadox Hemashield Platinum 4 Branch Graft; Boston Scientific Corporation, Natick, Mass) was sewn with a 5-0 Prolene running suture for supracommissural ascending aortic replacement (Figure 1). The proximal graft anastomosis was made with a 5-0 Prolene running suture. There was a change in clamp site before we could manage the distal graft anastomosis. One clamp was set on the innominate artery at the base of vessel and another clamp was set on the aorta between the innominate artery and left carotid artery. The former clamp on the ascending aorta could be removed and the dissected aortic tissue proximal to the left carotid artery was resected. The distal anastomosis was made with a 5-0 Prolene running suture that exceeded the innominate artery to ensure a sufficient proximal landing zone.

After distal graft anastomosis was accomplished, the graft limbs were clamped separately and the aortic crossclamp removed (the innominate artery was still clamped). Thereafter, the left carotid artery was doubleclamped at the base of the vessel. The left carotid artery was cut between 2 clamps. The proximal end was sealed with a 5-0 Prolene suture; the distal end was anastomosed to the graft limb with a 5-0 Prolene running suture in an end-to-end manner. After the left carotid artery was attached to the graft limb (bilateral cerebral perfusion resumed), the rewarming process was begun. During the rewarming process, the left subclavian artery was cut and attached to the graft limbs with a 5-0 Prolene running suture in the same manner. Then, the innominate artery (already cut and clamped) was attached to the graft limb with a 5-0 Prolene suture. One 10-mm limb, which served as the antegrade delivery approach for the endovascular procedure, remained free. After the endograft procedure, the limb was ligated and sewn with a 4-0 Prolene suture.

\section{Endovascular Repair Portion (Aortic Arch)}

The proximal landing zone and length measurements were obtained by conducting an angiographic imaging study in the hybrid interventional operative suite equipped with a floor-mounted C-arm imaging system (Simens Medical, Forchheim, Germany). Antegrade access was obtained through the 10-mm side branch (Figure 1) of the 4-branch Dacron graft, which allowed the guidewire and catheter to be directed into the desired position, which helped to direct the delivery system (Figure 2). The diameter of the endograft for arch repair (Zenith; Cook Medical, Bloomington, Ind) and 4-branch Dacron graft are described in Table 1. The endograft length ranged from 186 to $207 \mathrm{~mm}$, which ensured the distal end of the endograft could be located in the descending aorta.

\section{Migration and Endoleak}

A device migration was defined as cranial or caudal movement of the proximal or distal component of the device in excess of $5 \mathrm{~mm}$ with respect to a fixed landmark. Angiographic and computed tomography angiography (CTA) studies were performed to determine endoleak during the operation and follow-up period, respectively.

\section{Fate of False Lumen}

All CTA scans were reviewed by 2 physicians, and discordant results were adjudicated by a third physician. The preoperative and discharge diagnostic CTA scans were used as a baseline for thrombosis formation in false lumen and true lumen expansion, respectively.

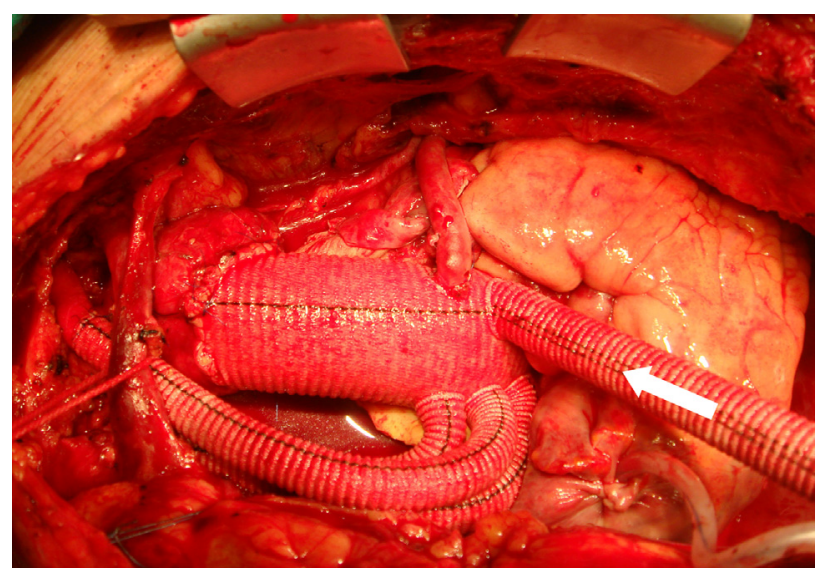

FIGURE 1. Ascending aortic replacement with a 4-branch Dacron graft. Three branches were connected to the innominate artery, the left common carotid artery, and the left subclavian artery. The perfusion branch $(10 \mathrm{~mm})$ acted as antegrade delivery approach (arrow).

\section{Statistical Analysis}

Results are expressed as mean \pm SD or median and range unless otherwise specified. Time-related survival rate was estimated with KaplanMeier analysis. Changes in diameters of true lumen were tested with the paired $t$ test. A value of $P<.05$ was considered statistically significant. Statistical analyses were performed with SPSS 17.0 software (SPSS, Inc, Chicago, Ill).

\section{RESULTS}

From November 2009 to April 2011, twenty-one consecutive patients who met previously defined criteria for acute type A aortic dissection were enrolled in the study. Seventeen of 21 patients $(81 \%)$ were male and 4 of $21(19 \%)$ were female. Mean age was $61.5 \pm 6.7$ years. Preoperative comorbidities of the patients are listed in Table 2.

No deep hypothermic circulatory arrest and selective cerebral perfusion were required. Mean crossclamp time was $46.8 \pm 12.1$ minutes and mean cardiopulmonary bypass time was $139.1 \pm 29.2$ minutes. The average procedure duration combining open and endovascular repair was $6.8 \pm$ 0.9 hours. Operative data are presented in Table 1.

The median length of stay in the intensive care unit after the procedure was 2 days (range, 1-12 days). Major postoperative events are listed in Table 3 . One of 3 patients with preoperative acute renal dysfunction required continuous renal replacement therapy. Two cases of neurologic events $(9.5 \%)$ were observed in this cohort. One patent had a transient neurologic deficit that occurred during the intensive care unit stay; the other had cerebral hemorrhage confirmed by cerebral computed tomographic scan during follow-up.

\section{Mortality}

The in-hospital mortality rate was $4.8 \%(1 / 21)$. One patient who had acute renal dysfunction before surgery died of postoperative complications from multiple-system and organ failure. Follow-up ranged from 3 to 21 months, with 


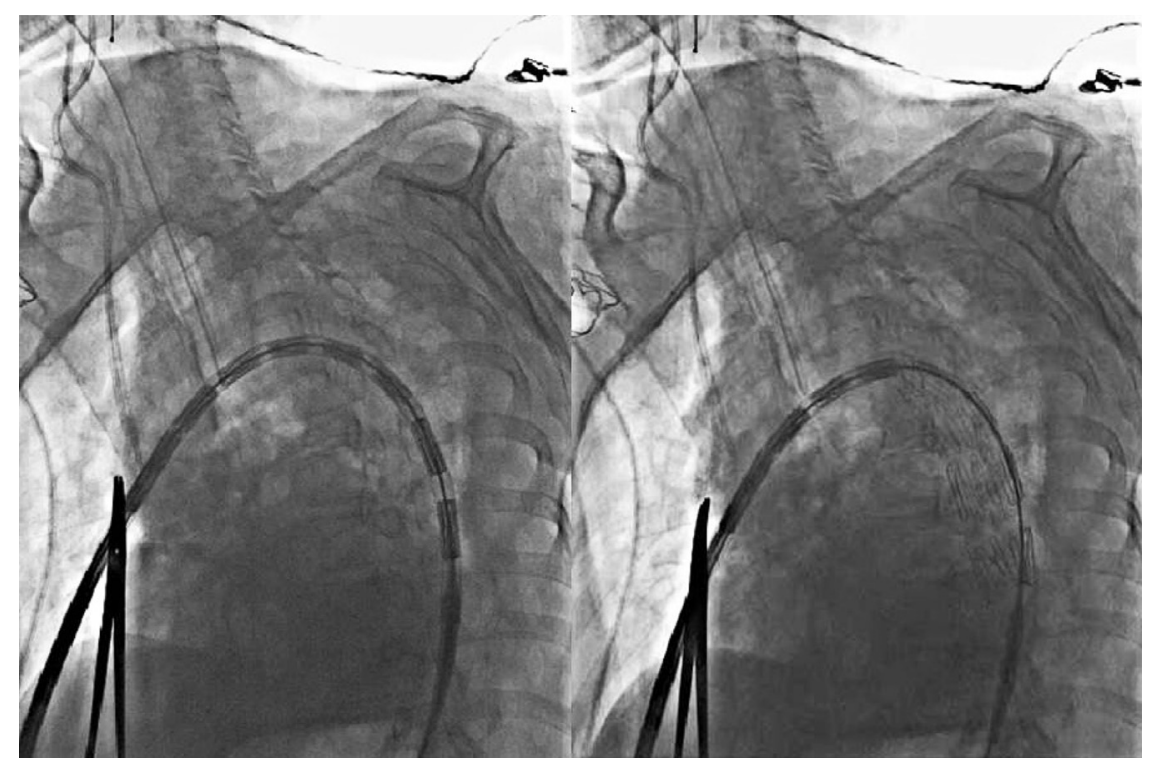

FIGURE 2. Antegrade guidewire positioning and deployment of an endograft via the branch of the Dacron graft.

a mean of $13.8 \pm 6.1$ months. No patient was lost to followup. The overall Kaplan-Meier 1-month survival rate was $95.2 \%$ (95\% confidence interval, $86.2 \%-100 \%)$; the 12 -month survival rate was $90.5 \%$ (95\% confidence interval, $78.0 \%-100 \%$; Figure 3).

\section{Migration and Endoleak}

There was no caudal migration of endovascular graft observed in the discharge and follow-up CTA scan. Type I endoleak occurred in 1 patient $(4.8 \%)$ during the operation and was treated successfully treated with a TBE36-71 cuff (Cook Medical) (Figure 4). The angiographic study performed during the operation and the postoperative CTA scan double-confirmed that the endoleak was cured.

\section{TABLE 1. Operative data}

\begin{tabular}{lrr}
\hline \multicolumn{1}{c}{ Operative variable } & n & \multicolumn{1}{c}{$\%$} \\
\hline Axillary cannulation & 21 & 100.0 \\
Femoral cannulation & 17 & 81.0 \\
Bentall & 1 & 4.8 \\
Ascending aortic replacement & 20 & 95.2 \\
Commissure sling & 5 & 23.8 \\
Sinus of Valsalva plasty & 6 & 28.6 \\
CABG & 2 & 9.5 \\
Femoral-femoral artery bypass & 1 & 4.8 \\
Dacron graft diameter & & \\
$26 \mathrm{~mm}$ & 1 & 4.8 \\
$28 \mathrm{~mm}$ & 6 & 28.6 \\
$30 \mathrm{~mm}$ & 13 & 61.9 \\
$32 \mathrm{~mm}$ & 1 & \\
Stent graft diameter & & 71.4 \\
$32 \mathrm{~mm}$ & 6 & \\
$34 \mathrm{~mm}$ & 15 &
\end{tabular}

\section{Fate of False Lumen and True Lumen}

Complete thrombosis formation of a false lumen at the level of the left inferior pulmonary vein was observed in $85.0 \%(17 / 20)$ of all patients by discharge. True lumen expansion exceeding $5 \mathrm{~mm}$ was observed in $90.0 \%(18 / 20)$ of all patients at the level of the left inferior pulmonary vein. True lumen expansion at different levels are indicated in Table 4.

\section{DISCUSSION}

The conventional approach for acute Stanford type A aortic dissection is a ascending aortic replacement and hemi-arch replacement, which can be accomplished in an emergency situation with acceptable in-hospital mortality risk. The current study represents a pilot cohort of a 1-stage hybrid total arch repair approach that combines open

TABLE 2. Preoperative comorbidities

\begin{tabular}{lrr}
\hline \multicolumn{1}{c}{ Comorbidity } & $\mathbf{n}$ & \multicolumn{1}{c}{$\%$} \\
\hline Cerebral infarction & 1 & 4.8 \\
Coronary artery disease & 4 & 19.0 \\
Hypertension & 16 & 76.2 \\
Diabetes mellitus & 1 & 4.8 \\
Atrial fibrillation & 1 & 4.8 \\
Acute renal dysfunction & 3 & 14.3 \\
Pericardial tamponade & 2 & 9.5 \\
COPD & 1 & 4.8 \\
Aortic regurgitation & & \\
$\quad$ None & 2 & 9.5 \\
Mild & 8 & 38.1 \\
Moderate & 8 & 14.3 \\
Severe & 3 & \\
COPD, Chronic obstructive pulmonary disease. & &
\end{tabular}


TABLE 3. Postoperative events

\begin{tabular}{lrr}
\hline \multicolumn{1}{c}{ Event } & n & $\%$ \\
\hline Ventilation & & \\
$\quad<24 \mathrm{~h}$ & 13 & 61.9 \\
$\geq 24 \mathrm{~h}$ and $<48 \mathrm{~h}$ & 4 & 19.0 \\
$\geq 48 \mathrm{~h}$ and $<72 \mathrm{~h}$ & 0 & 0.0 \\
$\geq 72 \mathrm{~h}$ & 4 & 19.0 \\
Tracheostomy & 1 & 4.8 \\
Acute myocardial infarction & 0 & 0.0 \\
Acute renal failure & 1 & 4.8 \\
Transient neurologic deficit & 1 & 4.8 \\
Stroke & 0 & 0.0 \\
Paralysis & 0 & 0.0 \\
Reexploration & 1 & 4.8 \\
Sternal infection & 0 & 0.0 \\
\hline
\end{tabular}

ascending aortic and hemi-arch replacement and endovascular total arch repair without deep hypothermic circulatory arrest in acute Stanford type A aortic dissection.

Rationale for adding the total arch repair and the endovascular stent graft in the descending aorta was based primarily on 2 points: (1) to minimize the risk of secondary enlargement of the residual false lumen in the arch and descending aorta and (2) to facilitate a potential second-stage open or endovascular thoracoabdominal aortic repair. ${ }^{9}$ Various hybrid procedures combining conventional total arch repair under deep hypothermic circulatory arrest and open/percutaneous distal stent placement have been reported. $^{7,10,11}$ In our previously reported cohort of 107 patients who underwent total arch replacement and a stented elephant trunk procedure for acute Stanford type A aortic dissection, the rate of thrombus formation in false lumen was observed in $95 \%$ of patients at the descending aorta level (around the elephant trunk graft) and in $69 \%$ of patients at the diaphragm level. ${ }^{12}$ Gorlitzer and colleagues ${ }^{13}$ reported a similar result-complete thrombus formation of the false lumen in the perigraft space in the stented segment of the prosthesis in $86 \%$ of 14 patients within 2 weeks after hybrid surgery. The results of current study support the high prevalence of early thrombosis in false lumen in the descending aorta by inserting a stent graft into true lumen of the arch and the descending aorta. The thrombosis of the false lumen was recognized to be of potential benefit. The formation of thrombosis in the false lumen might result in eliminating possible secondary tear sites in the aortic arch and the descending aorta, preventing secondary enlargement. Spinal cord injury resulting from insufficient segmental blood perfusion induced by thrombosis of the false lumen in the descending aorta is a potential risk after inserting a stent graft into the arch and the descending aorta despite the absence of such cases in this cohort.

True lumen expansion in the abdominal aorta is another notable finding in the current study (Figure 4). Significant expansion of the true lumen at 4 defined levels (Table 4) during follow-up indicated the fact that insertion of the stent graft into the arch and the descending aorta led to a beneficial rebalancing of blood flow between the true lumen and the patent false lumen in the distal descending aorta and abdominal aorta. The expansion of the true lumen and pressure reduction in the false lumen in the abdominal aorta could result in preferable organ and lower limb perfusion, and aortic remodeling in the abdominal aorta.

Deep hypothermic circulatory arrest had been associated with significant morbidity and mortality for patients with multiple comorbidities and in elderly patients. ${ }^{11,14,15}$ Deep hypothermic circulatory arrest is considered to be a double-edged sword. With the help of hypothermic circulatory arrest, open arch repair can be performed as a routine procedure in many aortic centers. On the other hand, the

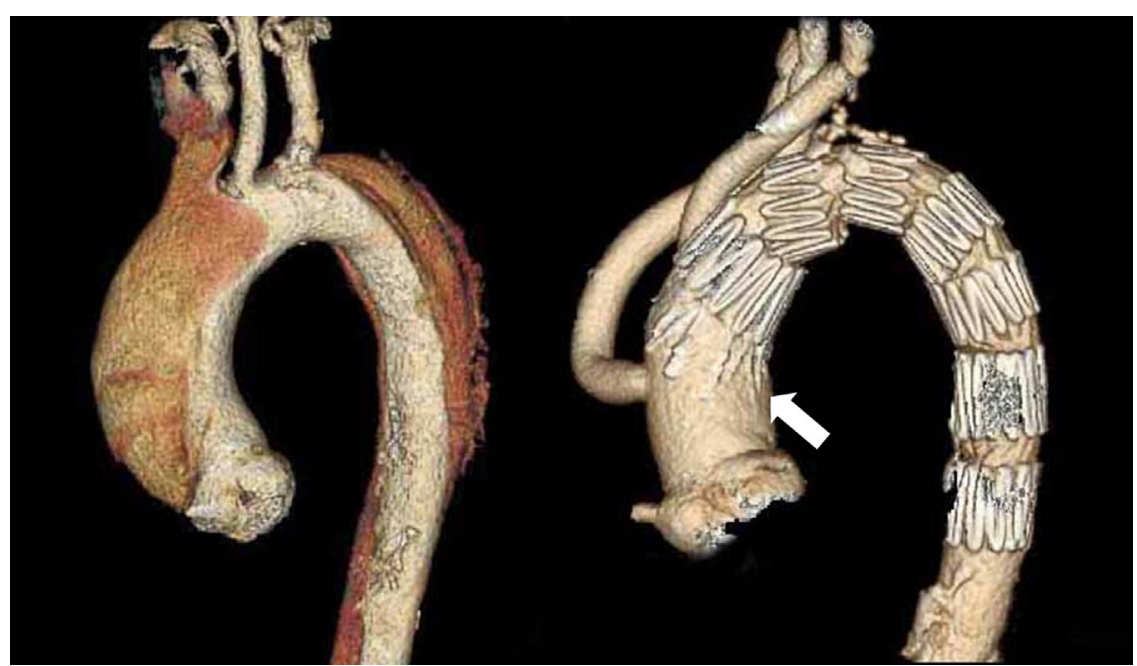

FIGURE 3. Preoperative (left) and postoperative (right) computed tomographic scan and 3-dimensional reconstruction. The patient with type I endoleak was treated successfully with a cuff (arrow) during the operation. 


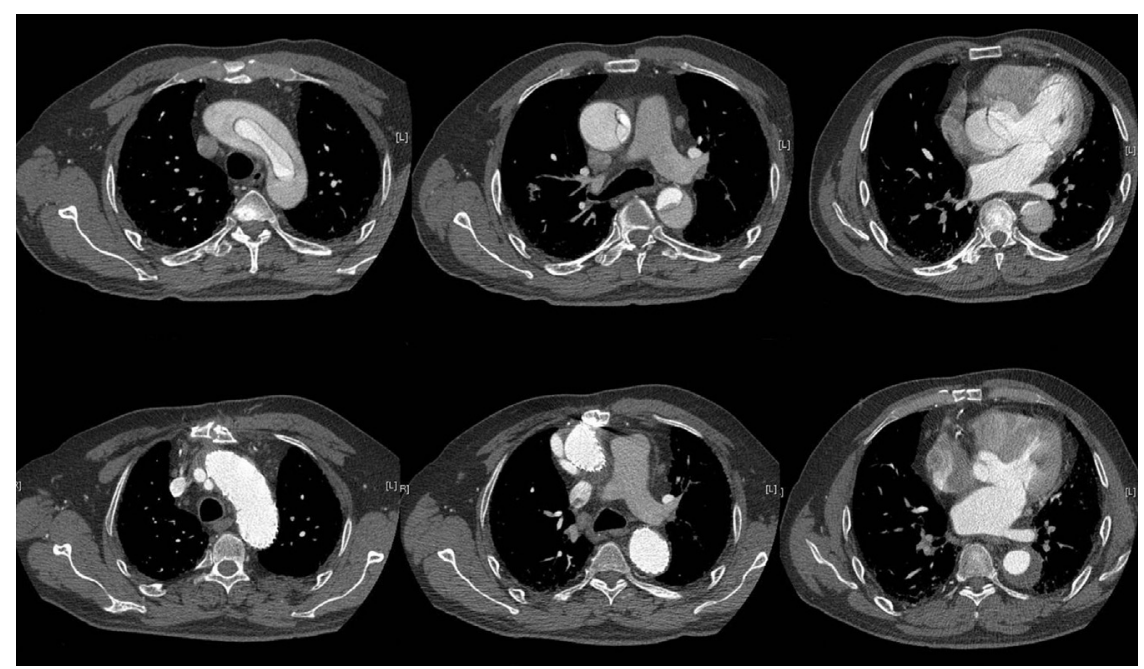

FIGURE 4. Cross-sectional computed tomographic scans during follow-up indicated thrombosis formation and expansion of the true lumen in the descending aorta. Upper row, Preoperative computed tomographic scan. Lower row, Postoperative computed tomographic scan.

process of deep hypothermic circulatory arrest can cause focal and/or global cerebral injury resulting from provocation of an inflammatory response and debris during manipulation. Blood-brain barrier damage has been observed in a rabbit model of deep hypothermic circulatory arrest. ${ }^{16}$ The avoidance of deep hypothermic circulatory arrest might, theoretically, provide neurologic benefit for patients with acute type A aortic dissection. We found in the current study that the in-hospital mortality was low and was coupled with a low rate of neurologic and other adverse events.

The potential proximal landing zone for endovascular repair in patients with Stanford type A dissection was diminished. Reconstruction of the ascending aorta provided the possible landing zone for the endovascular graft for total arch repair. The preferable result of stent migration and endoleak indicated that 4-mm oversizing of the stent to graft could provide a sufficient landing in such cases. Composite aortic root replacement was not common in this patient

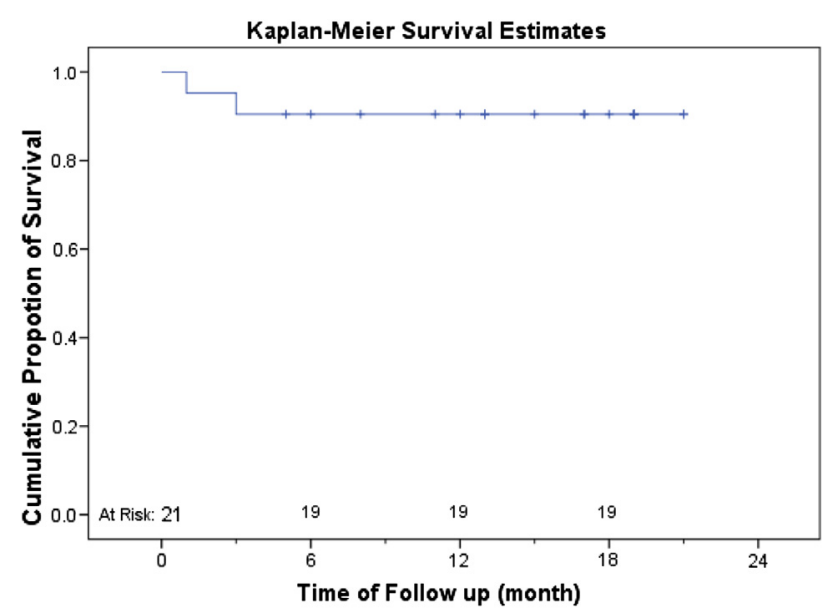

FIGURE 5. Kaplan-Meier analysis of time-related survival function. cohort because most of the patients with aortic regurgitation in current study group could be repaired successfully. In addition, a bioglue was not used in the entire group because the dissected aortic wall in the sinus of Valsalva was replaced with a patch of Dacron graft.

All procedures in the current study were performed in a hybrid operation room equipped with a universal floormounted angiographic C-arm system in one stage. In patients with acute Stanford type A aortic dissection, the hemodynamic status in residual false lumen of arch and distal aorta would change significantly after open repair stage of hybrid total arch repair procedure. Risk of rupture of untreated aorta would increase the mortality rate substantially during the period between the open repair portion and the endovascular portion of the hybrid operation. ${ }^{5}$ The open repair portion and the endovascular portion of the hybrid procedure should be performed in 1 stage, instead of staged manner, whenever possible. Antegrade delivery of the endograft was used as the primary approach in current study for the endovascular procedure, which avoided an additional femoral artery incision.

Limitations to this study should be noted. In general, the result of current study might contain bias because of the relatively small study population and the observational nature of the research type. There was no direct evidence for

TABLE 4. Diameter expansion of the true lumen at different levels

\begin{tabular}{lcccc}
\hline \multicolumn{1}{c}{ Level } & $\begin{array}{c}\text { Preoperative, } \\
\text { mm }\end{array}$ & $\begin{array}{c}\text { Postoperative, } \\
\text { mm }\end{array}$ & $\begin{array}{c}\text { Change, } \\
\text { mm }\end{array}$ & $\begin{array}{c}\boldsymbol{P} \\
\text { value }\end{array}$ \\
\hline Aortic arch & $18.7 \pm 4.0$ & $26.8 \pm 3.6$ & $8.1 \pm 4.8$ & $<.001$ \\
Carina of trachea & $12.6 \pm 3.8$ & $26.5 \pm 3.5$ & $13.9 \pm 4.8$ & $<.001$ \\
$\begin{array}{l}\text { Left inferior } \\
\quad \text { pulmonary vein }\end{array}$ & $10.7 \pm 3.6$ & $21.5 \pm 5.6$ & $10.8 \pm 3.8$ & $<.001$ \\
Celiac artery & $11.3 \pm 4.2$ & $14.2 \pm 3.1$ & $2.9 \pm 3.8$ & .37 \\
\hline
\end{tabular}

Data are measured as mean \pm standard deviation. 
superior outcome over the standard approach. The exact conclusions of advantages over conventional approaches need to be supported by further investigations. Technically, the durability of the endograft should be considered when this hybrid procedure is offered to younger patients because younger patients have a longer life expectancy and greater chance of secondary thoracoabdominal aortic repair resulting from their pathogenesis. Furthermore, the reliable fixation of the endograft in the current study was obtained by sufficient oversizing. ${ }^{11,17}$ The possibility of distal rupture in the descending aorta caused by oversizing the endograft, and long-term fixation of the endograft need further investigation.

\section{CONCLUSIONS}

Hybrid total arch repair without deep hypothermic circulatory arrest offers a promising method to reduce (1) the risk of mortality and morbidity during the postoperative period and (2) late adverse events resulting from false lumen enlargement within the arch and the descending aorta. This procedure could be a step forward in treating the aortic arch and descending aorta in acute Stanford type A aortic dissection.

\section{References}

1. Kallenbach K, Oelze T, Salcher R, Hagl C, Karck M, Leyh RG, et al. Evolving strategies for treatment of acute aortic dissection type A. Circulation. 2004;110: II-243-9.

2. Mimoun L, Detaint D, Hamroun D, Arnoult F, Delorme G, Gautier M, et al. Dissection in Marfan syndrome: the importance of the descending aorta. Eur Heart J. 2011;32:443-9.

3. Zierer A, Voeller RK, Hill KE, Kouchoukos NT, Damiano RJ Jr, Moon MR. Aortic enlargement and late reoperation after repair of acute type A aortic dissection. Ann Thorac Surg. 2007;84:479-87.

4. Kato M, Kuratani T, Kaneko M, Kyo S, Ohnishi K. The results of total arch graft implantation with open stent-graft placement for type A aortic dissection. J Thorac Cardiovasc Surg. 2002;124:531-40.
5. Marullo AGM, Bichi S, Pennetta RA, Di Matteo G, Cricco AM, Specchia L, et al. Hybrid aortic arch debranching with staged endovascular completion in DeBakey type I aortic dissection. Ann Thorac Surg. 2010;90:1847-53.

6. Westaby S, Saito S, Katsumata T. Acute type A dissection: conservative methods provide consistently low mortality. Ann Thorac Surg. 2002;73:707-13.

7. Uchida N, Shibamura H, Katayama A, Shimada N, Sutoh M, Ishihara H. Operative strategy for acute type A aortic dissection: ascending aortic or hemiarch versus total arch replacement with frozen elephant trunk. Ann Thorac Surg. 2009;87:773-7.

8. Tsagakis K, Pacini D, Di Bartolomeo R, Gorlitzer M, Weiss G, Grabenwoger M, et al. Multicenter early experience with extended aortic repair in acute aortic dissection: is simultaneous descending stent grafting justified? J Thorac Cardiovasc Surg. 2010;140:S116-20.

9. Sun L, Qi R, Chang Q, Zhu J, Liu Y, Yu C, et al. Surgery for Marfan patients with acute type A dissection using a stented elephant trunk procedure. Ann Thorac Surg. 2008;86:1821-5.

10. Pochettino A, Brinkman WT, Moeller P, Szeto WY, Moser W, Cornelius K, et al. Antegrade thoracic stent grafting during repair of acute DeBakey I dissection prevents development of thoracoabdominal aortic aneurysms. Ann Thorac Surg. 2009;88:482-90.

11. Milewski RK, Szeto WY, Pochettino A, Moser GW, Moeller P, Bavaria JE. Have hybrid procedures replaced open aortic arch reconstruction in highrisk patients? A comparative study of elective open arch debranching with endovascular stent graft placement and conventional elective open total and distal aortic arch reconstruction. J Thorac Cardiovasc Surg. 2010; 140:590-7.

12. Sun L-Z, Qi R-D, Chang Q, Zhu J-M, Liu Y-M, Yu C-T, et al. Surgery for acute type A dissection using total arch replacement combined with stented elephant trunk implantation: experience with 107 patients. J Thorac Cardiovasc Surg. 2009;138:1358-62.

13. Gorlitzer M, Weiss G, Meinhart J, Waldenberger F, Thalmann M, Folkmann S, et al. Fate of the false lumen after combined surgical and endovascular repair treating stanford type A aortic dissections. Ann Thorac Surg. 2010;89:794-9.

14. Mehta RH, Suzuki T, Hagan PG, Bossone E, Gilon D, Llovet A, et al. Predicting death in patients with acute type A aortic dissection. Circulation. 2002;105: 200-6.

15. Lai DT, Robbins RC, Mitchell RS, Moore KA, Oyer PE, Shumway NE, et al. Does profound hypothermic circulatory arrest improve survival in patients with acute type A aortic dissection? Circulation. 2002;106:I-218-28.

16. Huang HB, Tian C, Liu L, Liu WZ, Chang Q. Expression of occludin in brain tissues after deep hypothermic circulatory arrest: Study in a rabbit model. $\mathrm{Mol}$ Cardiol China. 2009;9:155-7.

17. Greenberg RK, Haddad F, Svensson L, O’Neill S, Walker E, Lyden SP, et al. Hybrid approaches to thoracic aortic aneurysms: the role of endovascular elephant trunk completion. Circulation. 2005;112:2619-26. 